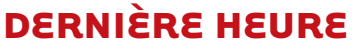

\section{Neuroprotection par l'activation des sirtuines dans des modèles simplifiés de chorée de Huntington}

J. Alex Parker, Margarita Arango, Salima Abderrahmane, Emmanuel Lambert, Cendrine Tourette, Hélène Catoire, Christian Néri

$>$ La chorée de Huntington est une maladie autosomique dominante caractérisée par des dysfonctionnements moteurs incontrôlables, accompagnés par une atteinte des fonctions cognitives et de l'humeur des patients. L'issue en est fatale entre 15 et 20 ans après l'apparition des premiers signes cliniques [1]. En France, 6000 personnes sont affectées. La maladie est causée par une expansion de triplets CAG, codant pour une glutamine, dans le gène ITI5 qui code pour la huntingtine. Chez les sujets non affectés, les répétitions s'échelonnent entre 6 et 35 triplets. Au-delà de 36, plus le nombre de répétitions est élevé plus la maladie se déclare tôt.

La huntingtine est une protéine polymorphe en polyglutamine avec une région riche en proline dans sa partie aminoterminale, sans homologie de séquence notable avec d'autres protéines. Sa fonction reste mal définie, mais elle interagit avec de nombreux partenaires. Elle a été impliquée dans la régulation de la transcription, le trafic et le transport axonal et semble nécessaire pour la neurogenèse et la survie neuronale chez les mammifères. La région comportant les expansions de polyglutamines (poly $($ ) semble induire des changements de conformation, rendant la protéine sensible à un clivage protéolytique et à la formation de fragments aminoterminaux. Ces produits de clivage ont une propension à former des inclusions intracellulaires, souvent nucléaires, bien que des agrégats situés dans d'autres portions de la cellule ne soient pas rares. Le rôle de ces agrégats est controversé [2]. Certains suggèrent qu'ils sont responsables de la toxicité $[3,4]$, alors que d'autres pensent qu'ils jouent un rôle protecteur pour enrayer la progression de la maladie $[5,6]$.

Un des grands mystères concernant la pathogénie de la chorée de Huntington est que - bien que l'expression de la huntingtine soit pratiquement ubiquitaire - la perte neuronale chez les individus affectés est restreinte aux ganglions de la base, en particulier le striatum et certaines régions corticales. Il est probable que la pathogénie de la chorée de Huntington soit liée à une perte de fonction de la huntingtine normale et à une augmentation de la toxicité liée aux extensions en polyglutamines, qui pourraient diminuer la fonction de certaines protéines. Ainsi, la huntingtine mutée interfère avec la transcription, soit en formant les inclusions nucléaires décrites ci-dessus, soit en séquestrant des facteurs de transcription comme $\mathrm{p} 53$, CBP (CREB-binding protein), SPI (specificity protein 1 ) et le coactivateur transcriptionel CA150 [7-10].

La découverte importante selon laquelle des mutations géniques uniques peuvent dramatiquement augmenter la durée de vie de plusieurs organismes modèles a montré que le vieillissement était un
Inserm, Projet Avenir, Laboratoire de Biologie génomique, IFR BrocaSainte Anne,

2 ter, rue d'Alésia, 75014 Paris, France. parker@broca.inserm.fr neri@broca.inserm.fr

phénomène plastique avec une composante génétique marquée [11]. On peut citer parmi ces gènes le facteur de transcription du groupe Forkhead, FOXO, et la NAD-dependent histone deacetylase Sir2 [12, 13]. La phosphorylation et la downregulation de FOXO jouent un rôle important dans la voie de transduction de l'insuline/IGF-1 (insulin-like growth factor-1). Quand la transduction de l'insuline est inhibée, FOXO déphosphorylé peut entrer dans le noyau où il active de nombreux gènes pour induire, dans certaines circonstances, la mort cellulaire ainsi qu'une résistance au stress, l'allongement de la durée de vie et la suppression de tumeurs [14]. F0X0 peut être phosphorylé par JNK [15] et désacétylaté par Sir2. Or, SIR2 augmente la longévité en déplaçant les réponses dépendantes de FOXO de la mort vers la survie cellulaire $[16,17]$. Ainsi, une activité accrue de SIRTl, codant pour la sirtuine, l'homologue chez les mammifères de Sir2, est neuroprotectrice [18].

Récemment, il a été montré que plusieurs polyphénols augmentaient l'affinité de Sir2 pour certains de ses substrats protéiques [19], vraisemblablement par un mécanisme allostérique [20]. Parmi ces composés, le resvératrol est un produit naturel dérivé du raisin (et présent dans le vin rouge). En combinant une approche génétique et pharmacologique, nous avons utilisé deux modèles de chorée de Huntington [21]. Le premier est fondé sur la surexpression 
ciblée de la huntingtine mutée aminoterminale dans les neurones sensibles au toucher du nématode Caenorhabditis elegans. Ce modèle exprime un dysfonctionnement neuronal puisque le ver répond beaucoup moins bien au toucher [22]. Le deuxième modèle est un peu plus proche de la maladie puisqu'il s'agit de neurones dérivés de striatum de souris avec des expansions CAG knocked-in dans le gène IT15 de souris [23]. Ces neurones exprimant la huntingtine mutée présentent un taux plus élevé de mort cellulaire. Dans les deux modèles, le resvératrol supprime les effets toxiques de la huntingtine mutée; les neurones de nématodes continuent à répondre au toucher et la mort neuronale est diminuée dans le modèle souris. $\varepsilon$ n revanche, l'activité du resvératrol est perdue chez des vers déficients pour Sir2 ou FOXO, et son effet protecteur est bloqué par des inhibiteurs de Sir2 sur les neurones de souris. Ces résultats suggèrent que la désacétylation de FOXO est liée à une survie maximale. FOXO induit

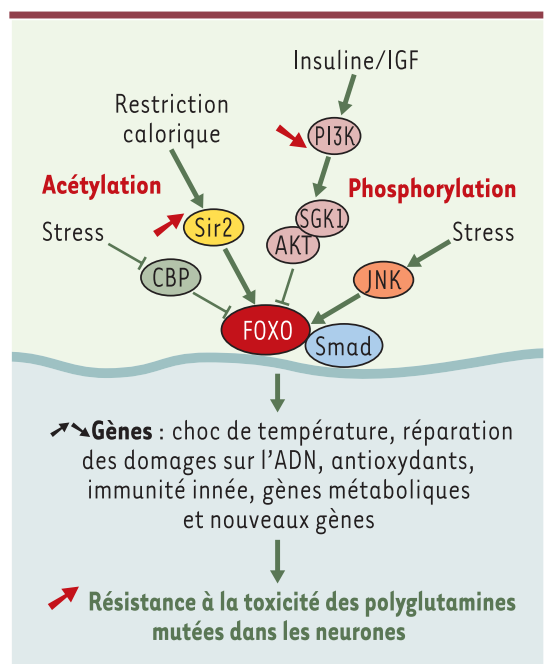

Figure 1. Les multiples voies de transduction et de transcription convergeant vers FOXO. De nombreuses voies de transduction convergent vers FOXO, comme la modification via l' acétylation par CBP et la désacétylation par Sir2. L'activité de FOXO est aussi modifiée à travers des phosphorylations par différentes kinases (AKT, SGKl et JNK). CBP: CREB-binding protein ; Sir2: silent information regulator 2; PI3K : phospho-inositide 3-kinase. l'expression de nombreux gènes, en particulier des protéines de choc thermique, ou impliquées dans la réparation de I'ADN, le contrôle de la croissance et de la mort cellulaire (Figure 1). La neuroprotection induite par SIRTI n'implique sans doute pas toutes les cibles transcriptionnelles de FOXO. La prochaine étape sera de déterminer quels gènes sont requis pour la neuroprotection, ce qui devrait donner naissance à un nouveau groupe de cibles thérapeutiques potentielles. La biodisponibilité du resvératrol n'étant pas bonne, d'autres analogues et activateurs de la sirtuine doivent être testés sur des modèles murins de chorée de Huntington. $\varepsilon$ n outre, un effet dans d'autres maladies neurodégénératives associées à une agrégation protéique n'est pas à négliger.

Enfin, une question importante concerne la nature des facteurs transcriptionnels conférant la résistance au stress et le maintien des fonctions neuronales: l'activation de sirtuine affecte-t-elle le trafic intracellulaire et/ou la transduction neuronale? Des études complémentaires permettront de répondre à cette question [24]. Quoi qu'il en soit, il est intéressant que des recherches dans le domaine du vieillissement ouvrent une nouvelle voie thérapeutique potentielle pour le traitement des maladies neurodégénératives. $\diamond$

Resveratrol rescues mutant

polyglutamine cytotoxicity

in nematode and mammalian neurons

\section{RÉFÉRENCES}

1. Landles C, Bates GP. Huntingtin and the molecular pathogenesis of Huntington's disease. Fourth in molecular medicine review series. EMBO Rep 2004; 5 : 958-63.

2. Sisodia SS. Nuclear inclusions in glutamine repeat disorders: are they pernicious, coincidental, or beneficial? Cell 1998; 95: 1-4.

3. Davies SW, Turmaine M, Cozens BA, et al. Formation of neuronal intranuclear inclusions underlies the neurological dysfunction in mice transgenic for the HD mutation. Cell 1997; $90:$ 537-48.

4. Difiglia M, Sapp $\varepsilon$, Chase KO, et al. Aggregation of huntingtin in neuronal intranuclear inclusions and dystrophic neurites in brain. Science 1997; 277 : 1990-3.

5. Arrasate M, Mitra S, Schweitzer ES, Segal MR,
Finkbeiner S. Inclusion body formation reduces levels of mutant huntingtin and the risk of neuronal death. Nature 2004; 431: 805-10.

6. Saudou F, Finkbeiner S, Devys D, Greenberg ME. Huntingtin acts in the nucleus to induce apoptosis but death does not correlate with the formation of intranuclear inclusions. Cell 1998; 95: 55-66.

7. Dunah AW, Jeong H, Griffin A, et al. Spl and TAFIII30 transcriptional activity disrupted in early Huntington's disease. Science 2002; 296: 2238-43.

8. Holbert S, Denghien I, Kiechle T, et al. The GIn-Ala repeat transcriptional activator CA150 interacts with huntingtin: neuropathologic and genetic evidence for a role in Huntington's disease pathogenesis. Proc Natl Acad Sci USA 2001; 98: 1811-6.

9. Steffan JS, Kazantsev A, Spasic-Boskovic 0, et al. The Huntington's disease protein interacts with $p 53$ and CREB-binding protein and represses transcription. Proc Natl Acad Sci USA 2000; 97: 6763-8.

10. Sugars KL, Rubinsztein DC. Transcriptional abnormalities in Huntington disease. Trends Genet 2003; 19: 233-8.

11. Kenyon C. The plasticity of aging: insights from longlived mutants. Cell 2005; 120: 449-60.

12. Hekimi S, Guarente L. Genetics and the specificity of the aging process. Science 2003; 299: 1351-4.

13. Tatar M, Bartke A, Antebi A. The endocrine regulation of aging by insulin-like signals. Science 2003; 299 : 1346-51.

14. Brunet $A$. Les multiples actions des facteurs de transcription FOXO. Med Sci (Paris) 2004; $20: 856-9$.

15. Wang MC, Bohmann D, Jasper H. JNK Extends life span and limits growth by antagonizing cellular and organism-wide responses to insulin signaling. Cell 2005; 121: 115-25.

16. Brunet A, Sweeney LB, Sturgill FJ, et al. Stressdependent regulation of FOXO transcription factors by the SIRT1 deacetylase. Science 2004; 303: 2011-5.

17. Motta MC, Divecha N, Lemieux M, et al. Mammalian SIRTl represses forkhead transcription factors. Cell 2004; 116: 551-63.

18. Araki T, Sasaki Y, Milbrandt, J. Increased nuclear NAD biosynthesis and SIRTl activation prevent axonal degeneration. Science 2004; 305: 1010-3.

19. Howitz KT, Bitterman KJ, Cohen HY, et al. Small molecule activators of sirtuins extend Saccharomyces cerevisiae lifespan. Nature 2003; 425: 191-6.

20. Kaeberlein M, McDonagh $\mathrm{T}$, Heltweg $B$, et al. Substrate specific activation fo sirtuins by resveratrol. J Biol Chem 2005 (sous presse).

21. Parker JA, Arango M, Abderrahmane S, et al. Resveratrol rescues mutant polyglutamine cytotoxicity in nematode and mammalian neurons. Nat Genet 2005; 37 : 349-50.

22. Parker JA, Connolly JB, Wellington C, et al. Expanded polyglutamines in Caenorhabditis elegans cause axonal abnormalities and severe dysfunction of PLM mechanosensory neurons without cell death. Proc Natl Acad Sci USA 2001; 98: 13318-23.

23. Trettel F, Rigamonti D, Hilditch-Maguire $P$, et al. Dominant phenotypes produced by the HD mutation in STHdh(Q111) striatal cells. Hum Mol Genet 2000; 9: 2799-809.

24. O'Hagan R, Chalfie M, Goodman MB. The MEC-4 $D \varepsilon G / \varepsilon N a C$ channel of Caenorhabditis elegans touch receptor neurons transduces mechanical signals. Nat Neurosci 2005; 8: 43-50. 